IMA Journal of Numerical Analysis (2009) 29, 158-179

doi:10.1093/imanum/drm044

Advance Access publication on March 11, 2008

\title{
Sparse convolution quadrature for time domain boundary integral formulations of the wave equation
}

\author{
W. HACKBUSCH AND W. KRESS $\dagger$ \\ Max Planck Institute for Mathematics in the Sciences, Inselstrasse 22, \\ D-04103 Leipzig, Germany \\ AND \\ S. A. SAUTER \\ Institut für Mathematik, Universität Zürich, Winterthurerstrasse 190, \\ CH-8057 Zürich, Switzerland
}

[Received on 15 December 2005; revised on 27 September 2006]

\begin{abstract}
Many important physical applications are governed by the wave equation. The formulation as time domain boundary integral equations involves retarded potentials. For the numerical solution of this problem, we employ the convolution quadrature method for the discretization in time and the Galerkin boundary element method for the space discretization. We introduce a simple a priori cut-off strategy where small entries of the system matrices are replaced by zero. The threshold for the cut-off is determined by an $a$ priori analysis which will be developed in this paper. This analysis will also allow to estimate the effect of additional perturbations such as panel clustering and numerical integration on the overall discretization error. This method reduces the storage complexity for time domain integral equations from $\mathrm{O}\left(M^{2} N\right)$ to $\mathrm{O}\left(M^{2} N^{\frac{1}{2}} \log M\right)$, where $N$ denotes the number of time steps and $M$ is the dimension of the boundary element space.
\end{abstract}

Keywords: retarded potentials; convolution quadrature; boundary element method; sparse representation.

\section{Introduction}

Boundary-value problems governed by the wave equation

$$
\partial_{t}^{2} u-\Delta u=f
$$

arise in many physical applications such as electromagnetic wave propagation or the computation of transient acoustic waves. Since such problems are typically formulated in unbounded domains, the method of integral equations is an elegant tool to transform this partial differential equation to an integral equation on the bounded surface of the scatterer.

Although this approach goes back to the early 1960s (cf. Friedman \& Shaw, 1962), the development of fast numerical methods for integral equations in the field of hyperbolic problems is still in its infancies compared to the vast of fast methods for elliptic boundary integral equations (cf. Sauter \& Schwab, 2004, and references therein). Existing numerical discretization methods include collocation methods with some stabilization techniques (cf. Birgisson et al., 1999; Bluck \& Walker, 1996; Davies, 1994, 1997;

\footnotetext{
†Email: kress@mis.mpg.de
} 
Davies \& Duncan, 2004; Miller, 1987; Rynne \& Smith, 1990) and Laplace-Fourier methods coupled with Galerkin boundary elements in space (Bamberger \& Ha-Duong, 1986; Costabel, 1994; Ding et al., 1989; Ha-Duong, 2003). Numerical experiments can be found, e.g. in Ha-Duong et al. (2003).

In Ergin et al. (2000), a fast version of the 'marching-on-in-time' (MOT) method is presented which is based on a suitable plane wave expansion of the arising potential. The advantage of this approach is that the computational complexity is reduced to $\mathrm{O}(N M)$ operations. However, the class of applications for which MOT is applicable is smaller compared to the direct discretization of the retarded boundary integral equations, e.g. the sources of the incoming waves have to be properly separated from the scatterer 'and' the observation points and the signals must be bandlimited. In addition, a stability and convergence analysis which takes into account the effect of the various perturbations (such as plane wave expansions) on the 'overall' discretization error is not available in a rigorous mathematical way.

We here employ the convolution quadrature method for the time discretization and a Galerkin boundary element method in space. The convolution quadrature method for the time discretization has been developed in Lubich (1988a,b, 1994) and Lubich \& Schneider (1992). It provides a straightforward way to obtain a stable time-stepping scheme using the Laplace transform of the kernel function. A straightforward implementation results in an algorithm with a storage complexity of $\mathrm{O}\left(N M^{2}\right)$ and a computational complexity of $\mathrm{O}\left(N^{2} M^{2}\right)$. In Hairer et al. (1985), FFT techniques have been introduced where the computational complexity is reduced to $\mathrm{O}\left(N \log ^{2} N M^{2}\right)$, while the storage costs stay unchanged.

Note that also the classical Galerkin discretization of the retarded boundary integral equations (see Bamberger \& Ha-Duong, 1986; Ha-Duong, 2003) leads to a block Töplitz system matrix where the matrix blocks $\mathbf{A}_{j}, 0 \leqslant j \leqslant N$, are of size $M \times M$ and sparse. More precisely, the number of nonzero entries in the system matrix $\mathbf{A}$ is, for piecewise constant boundary elements, of order $\mathrm{O}\left(M^{2}\right)$ and, for piecewise linear boundary elements, of order $\mathrm{O}\left(M^{2+\frac{1}{8}}\right)$ for this approach. The total cost for the computation of a full Galerkin approximation by using this approach sums up for piecewise constant boundary elements to $\mathrm{O}\left(M^{2} N\right)$ operations, while the operation count for piecewise linear boundary elements is $\mathrm{O}\left(N^{2} M^{3 / 2}\right)$. A drawback of this approach, however, is that the numerical integrations for computing the coefficients of the system matrix have to be carried out on the intersections of the boundary element mesh with the discrete light cone. The stable handling of these intersections and the implementation is especially complicated for curved panels.

In this paper, we introduce an alternative approach which is based on sparse matrix approximation. We introduce a simple cut-off strategy which reduces the computational costs to $\mathrm{O}\left(N^{\frac{3}{2}} M^{2} \log M\right)$, while the focus is on the storage consumptions which are reduced to $\mathrm{O}\left(N^{\frac{1}{2}} M^{2} \log M\right)$. Note that this approach has been extended in Kress \& Sauter (2006), where the computational complexity is reduced to $\mathrm{O}\left(N^{9 / 2} \log ^{11} M\right)$ and the storage cost to $\mathrm{O}\left(N^{7 / 2} \log ^{11} M\right)$. We emphasize that this analysis only shows the 'asymptotic' behaviour of the complexity. For practical problems, the leading constants behind these $\mathrm{O}(\cdot)$ estimates are essential and we are currently starting to implement our approach in order to compare the different approaches for practical problem sizes.

In Tables 1 and 2, the asymptotic complexity of these methods is depicted. Note that the error analysis will show that the relation $N \approx M^{m / 4+3 / 8}$ between the number of time steps $N$ and the dimension $M$ of the boundary element space is balancing the spatial and temporal errors and we employ this relation in both tables. As can be seen from these tables, our approach reduces the storage complexity more significantly while the FFT approach has a better computational performance in most cases. The direct Galerkin approximation of the retarded potentials also has a very good performance, while the drawbacks are the complicated numerical integration and the fact that a general perturbation analysis for the overall discretization is not available by now. 
TABLE 1 Storage requirements for the direct Galerkin discretization, the FFT approach, the sparse approximation by cut-off and the panel clustering method. The case $m=0$ corresponds to piecewise constant boundary elements, while $m=1$ indicates piecewise linear elements

\begin{tabular}{ccccc}
\hline & Direct Galerkin & FFT & Cut-off strategy & Panel clustering + cut-off \\
\hline$m=0$ & $\mathrm{O}\left(N M^{1+\frac{5}{8}}\right)$ & $\mathrm{O}\left(N M^{2}\right)$ & $\mathrm{O}\left(N M^{1+\frac{13}{16}} \log M\right)$ & $\mathrm{O}\left(N M^{1-\frac{1}{16}} \log ^{11} M\right)$ \\
$m=1$ & $\mathrm{O}\left(N M^{1+\frac{1}{2}}\right)$ & $\mathrm{O}\left(N M^{2}\right)$ & $\mathrm{O}\left(N M^{1+\frac{11}{16}} \log M\right)$ & $\mathrm{O}\left(N M^{1+\frac{9}{16}} \log ^{11} M\right)$ \\
\hline
\end{tabular}

TABLE 2 Computational complexity for the direct Galerkin approximation, the FFT approach, the sparse approximation by cut-off and the panel clustering method. Again, the case $m=0$ corresponds to piecewise constant boundary elements, while $m=1$ indicates piecewise linear elements

\begin{tabular}{ccccc}
\hline & Direct Galerkin & FFT & Cut-off strategy & Panel clustering + cut-off \\
\hline$m=0$ & $\mathrm{O}\left(N M^{2}\right)$ & $\mathrm{O}\left(N M^{2}\left(\log ^{2} N\right)\right)$ & $\mathrm{O}\left(N M^{2+\frac{3}{16}} \log M\right)$ & $\mathrm{O}\left(N M^{2-\frac{11}{16}} \log ^{11} M\right)$ \\
$m=1$ & $\mathrm{O}\left(N M^{2}\right)$ & $\mathrm{O}\left(N M^{2}\left(\log ^{2} N\right)\right)$ & $\mathrm{O}\left(N M^{2+\frac{5}{16}} \log M\right)$ & $\mathrm{O}\left(N M^{2+\frac{3}{16}} \log ^{11} M\right)$ \\
\hline
\end{tabular}

In this paper, we develop the theoretical framework for the analysis of the additional perturbations in the space discretization for the convolution quadrature approach.

The remainder of the paper is structured into five sections. In Section 2, we briefly introduce the formulation of the wave equation as an integral equation and recall its stability properties. Section 3 is devoted to the convolution quadrature method for the time discretization and the boundary element method for the space discretization. We introduce our a priori cut-off strategy to replace small matrix entries by zero and discuss some algorithmic aspects. In Section 4, we analyse the effect of the perturbation introduced by the cut-off strategy and prove the convergence of the corresponding solution. In Section 5, we discuss the complexity of our method. We show that the storage complexity in terms of the number $M$ of unknowns in space is reduced from $M^{2}$ to $N^{-\frac{1}{2}} M^{2}$. Finally, in Section 6, we summarize the results and give an outlook onto future research.

We emphasize that our simple cut-off strategy reduces the storage complexity of the method while the computational complexity is not reduced. However, this paper paves the way to introduce and analyse further perturbations in the space-time discretization. Forthcoming papers will be devoted to panel clustering techniques for the retarded potential boundary integral equation which will also reduce the dependence of the computational complexity on $M$ (cf. Hackbusch et al., 2007; Kress \& Sauter, 2006; Banjai \& Sauter, 2007).

\section{Integral formulation of the wave equation}

Let $\Omega \subset \mathbb{R}^{3}$ be a Lipschitz domain with boundary $\Gamma$. In this paper, we present efficient methods for numerically solving the homogeneous wave equation

$$
\partial_{t}^{2} u=\Delta u \quad \text { in } \Omega \times(0, T)
$$

with initial conditions

$$
u(\cdot, 0)=\partial_{t} u(\cdot, 0)=0 \quad \text { in } \Omega
$$


and boundary conditions

$$
u=g \quad \text { on } \Gamma \times(0, T),
$$

on a time interval $(0, T)$ for some $T>0$. For its solution, we employ an ansatz as a 'single-layer potential'

$$
u(x, t)=\int_{0}^{t} \int_{\Gamma} k(x-y, t-\tau) \phi(y, \tau) \mathrm{d} \Gamma_{y} \mathrm{~d} \tau, \quad(x, t) \in \Omega \times(0, T),
$$

where $k(z, t)$ is the fundamental solution of the wave equation,

$$
k(z, t)=\frac{\delta(t-\|z\|)}{4 \pi\|z\|},
$$

$\delta(t)$ being the Dirac delta distribution. The ansatz (2.2) satisfies the homogeneous equation (2.1a) and the initial conditions (2.1b). The extension $x \rightarrow \Gamma$ is continuous and hence, the unknown density $\phi$ in (2.2) is determined via the boundary conditions (2.1c), $u(x, t)=g(x, t)$. This results in the boundary integral equation for $\phi$ :

$$
(V \phi)(x, t):=\int_{0}^{t} \int_{\Gamma} k(x-y, t-\tau) \phi(y, \tau) \mathrm{d} \Gamma_{y} \mathrm{~d} \tau=g(x, t) \quad \forall(x, t) \in \Gamma \times(0, T) .
$$

Existence and uniqueness results for the solution of the continuous problem are proven in Lubich (1994). To recall them, we introduce appropriate norms and spaces. We define the Sobolev space $H^{s}(\Gamma), s \geqslant 0$, in the usual way (see, e.g. Hackbusch, 1992, or McLean, 2000). The range of $s$ for which $H^{s}(\Gamma)$ is defined may be limited, depending on the global smoothness of the surface $\Gamma$. Throughout, we let $[-k, k]$ denote the range of Sobolev indices for which $H^{s}(\Gamma)$ is defined with the negative-order spaces defined by duality in the usual way. The norm is denoted by $\|\cdot\|_{H^{s}(\Gamma)}$.

For real $r$ and $s \in[-k, k]$, the anisotropic Sobolev space $H^{r}\left(\mathbb{R} ; H^{s}(\Gamma)\right)$ is given by

$$
H^{r}\left(\mathbb{R} ; H^{s}(\Gamma)\right):=\left\{g: \Gamma \times \mathbb{R} \rightarrow \mathbb{R}: \int_{-\infty}^{\infty}(1+|\omega|)^{2 r}\|\mathscr{F} g(\cdot, \omega)\|_{H^{s}(\Gamma)}^{2} \mathrm{~d} \omega<\infty\right\},
$$

where $\mathscr{F}$ denotes the Fourier transform with respect to the time variable $t \in \mathbb{R}$. The norm in this space is given by

$$
\|f\|_{H^{r}\left(\mathbb{R} ; H^{s}(\Gamma)\right)}^{2}:=\int_{-\infty}^{\infty}(1+|\omega|)^{2 r}\|\mathscr{F} f(\cdot, \omega)\|_{H^{s}(\Gamma)}^{2} \mathrm{~d} \omega .
$$

The space $H_{0}^{r}\left(0, T ; H^{s}(\Gamma)\right)$ is defined by

$$
\begin{aligned}
H_{0}^{r}\left(0, T ; H^{s}(\Gamma)\right):= & \left\{g:[0, T] \times \Gamma \rightarrow \mathbb{R}: g=\left.g^{\star}\right|_{[0, T]}\right. \\
& \text { for some } \left.g^{\star} \in H^{r}\left(\mathbb{R}, H^{s}(\Gamma)\right) \text { with } g^{\star} \equiv 0 \text { on }\right]-\infty, 0[\}
\end{aligned}
$$

and the norm $\|\cdot\|_{H_{0}^{r}\left(0, T ; H^{s}(\Gamma)\right)}$ is given by

$$
\begin{aligned}
\|g\|_{H_{0}^{r}\left(0, T ; H^{s}(\Gamma)\right)}^{2}:= & \min \left\{\left\|g^{\star}\right\|_{H_{0}^{r}\left(\mathbb{R} ; H^{s}(\Gamma)\right)}: g^{\star} \in H^{r}\left(\mathbb{R}, H^{s}(\Gamma)\right)\right. \\
& \text { with } \left.g=\left.g^{\star}\right|_{[0, T]} \text { and } g^{\star} \equiv 0 \text { on }\right]-\infty, 0[\} .
\end{aligned}
$$


TheOREM 2.1 Let $g \in H^{r+2}\left(0, T ; H^{1 / 2}(\Gamma)\right)$ for some $r \in \mathbb{R}$. Then, (2.4) has a unique solution $\phi \in H^{r}\left(0, T ; H^{-1 / 2}(\Gamma)\right)$ with

$$
\|\phi\|_{H_{0}^{r}\left(0, T ; H^{-1 / 2}(\Gamma)\right)} \leqslant C_{T}\|g\|_{H_{0}^{r+2}\left(0, T ; H^{1 / 2}(\Gamma)\right)} .
$$

For $r>5 / 2$, the pointwise estimate

$$
\|\phi(\cdot, t)\|_{H^{-1 / 2}(\Gamma)} \leqslant C_{T}\|g\|_{H_{0}^{r+2}\left(0, T ; H^{1 / 2}(\Gamma)\right)}
$$

holds for all $t \in[0, T]$.

For a proof, we refer to Bamberger \& Ha-Duong (1986, Proposition 3), respectively, Lubich (1994, (2.23), (2.24)).

\section{Numerical discretization}

\subsection{Time discretization via convolution quadrature}

For the time discretization, we employ the convolution quadrature approach which has been developed by Lubich (1988a,b, 1994) and Lubich \& Schneider (1992). We do not recall the theoretical framework here but directly apply the approach to the wave equation.

We split the time interval $[0, T]$ into $N+1$ time steps of equal length $\Delta t=T / N$ and compute an approximate solution at the discrete time levels $t_{n}=n \Delta t$. The continuous convolution operator $V$ is replaced by the discrete convolution operator

$$
\left(V^{\Delta t} \phi_{\Delta t}\right)_{n}(x):=\sum_{j=0}^{n} \int_{\Gamma} \omega_{n-j}^{\Delta t}(x-y) \phi_{\Delta t}^{j}(y) \mathrm{d} \Gamma_{y},
$$

for $n=1, \ldots, N$. The convolution weights $\omega_{n}^{\Delta t}(x)$ will be defined below (see (3.6)). The semidiscrete problem is given by

$$
\left(V^{\Delta t} \phi_{\Delta t}\right)_{n}(x)=g_{\Delta t}^{n}(x), \quad n=1, \ldots, N, \quad x \in \Gamma,
$$

where $g_{\Delta t}^{n}(x)$ is some approximation to $g\left(x, t_{n}\right)$, or $g\left(x, t_{n}\right)$ itself.

Following the approach in Lubich (1988a,b, 1994), the convolution quadrature method is based on a linear multistep method which, for an ordinary differential equation $u^{\prime}(t)=f(u(t))$, can be formulated as

$$
\sum_{j=0}^{k} \alpha_{j} u^{n+j-k}=\Delta t \sum_{j=0}^{k} \beta_{j} f\left(u^{n+j-k}\right)
$$

where $u^{n} \approx u\left(t_{n}\right)$. Let

$$
\gamma(\zeta):=\frac{\sum_{j=0}^{k} \alpha_{j} \zeta^{k-j}}{\sum_{j=0}^{k} \beta_{j} \zeta^{k-j}}
$$

be the quotient of the generating polynomials of the linear multistep method (3.3). 
Definition 3.1 The convolution weights $\omega_{n}^{\Delta t}(x-y)$ of the convolution quadrature method (3.2) are given by the coefficients of the power series of the Laplace transform $\hat{k}(z, \gamma(\zeta) / \Delta t)=(4 \pi\|z\|)^{-1}$ $\exp \left(-\frac{\gamma(\zeta)}{\Delta t}\|z\|\right)$ of $(2.3)$, i.e.

$$
\hat{k}\left(z, \frac{\gamma(\zeta)}{\Delta t}\right)=\sum_{n=0}^{\infty} \omega_{n}^{\Delta t}(z) \zeta^{n}
$$

We employ the second-order accurate, $A$-stable BDF2 scheme which is given by

$$
\alpha_{0}^{\mathrm{BDF} 2}=\frac{1}{2}, \quad \alpha_{1}^{\mathrm{BDF} 2}=-2, \quad \alpha_{2}^{\mathrm{BDF} 2}=\frac{3}{2}, \quad \beta_{0}^{\mathrm{BDF} 2}=1,
$$

i.e.

$$
\gamma^{\mathrm{BDF} 2}(\zeta)=\frac{1}{2}\left(\zeta^{2}-4 \zeta+3\right)
$$

Because the kernel function only depends on the distance $d=\|x-y\|$, we write $\hat{k}(d, \cdot)$ and $\omega_{n}^{\Delta t}(d)$ short for $\hat{k}(x-y, \cdot)$ and $\omega_{n}^{\Delta t}(x-y)$. The coefficients of the power series (3.4) can be obtained by the Taylor expansion of $\hat{k}\left(d, \frac{\gamma(\zeta)}{\Delta t}\right)$ about $\zeta=0$,

$$
\omega_{n}^{\Delta t}(d)=\left.\frac{1}{n !} \frac{\partial^{n} \hat{k}\left(d, \frac{\gamma(\zeta)}{\Delta t}\right)}{\partial \zeta^{n}}\right|_{\zeta=0}=\left.\frac{1}{n !} \frac{1}{4 \pi d} \frac{\partial^{n} \mathrm{e}^{-\frac{\gamma(\zeta)}{\Delta t} d}}{\partial \zeta^{n}}\right|_{\zeta=0}
$$

Using the formula for multiple differentiation of composite functions (see, e.g. Gradshteyn \& Ryzhik, 1965), we obtain the explicit representation

$$
\omega_{n}^{\Delta t}(d)=\frac{1}{n !} \frac{1}{4 \pi d}\left(\frac{d}{2 \Delta t}\right)^{n / 2} \mathrm{e}^{-\frac{3 d}{2 \Delta t}} H_{n}\left(\sqrt{\frac{2 d}{\Delta t}}\right)
$$

where $H_{n}$ are the Hermite polynomials.

\subsection{Space discretization. Galerkin boundary element methods}

In Section 3.1, we have derived the semidiscrete problem: For $n=1,2, \ldots, N$, find $\phi_{\Delta t}^{n} \in H^{-1 / 2}(\Gamma)$ such that

$$
\sum_{j=0}^{n} \int_{\Gamma} \omega_{n-j}^{\Delta t}(x-y) \phi_{\Delta t}^{j}(y) \mathrm{d} \Gamma_{y}=g_{\Delta t}^{n}(x), \quad n=1, \ldots, N, \quad x \in \Gamma .
$$

For the space discretization, we employ a Galerkin boundary element method. Let $\mathscr{G}$ be a regular (in the sense of Ciarlet, 1987) boundary element mesh on $\Gamma$ consisting of shape regular, possibly curved triangles. For a triangle $\tau \in \mathscr{G}$, the (regular) pullback to the reference triangle $\widehat{\tau}:=\operatorname{conv}\left\{\left(\begin{array}{l}0 \\ 0\end{array}\right),\left(\begin{array}{l}1 \\ 0\end{array}\right),\left(\begin{array}{l}0 \\ 1\end{array}\right)\right\}$ is denoted by $\chi_{\tau}: \widehat{\tau} \rightarrow \tau$. The space of piecewise constant, discontinuous functions is

$$
S_{-1,0}:=\left\{u \in L^{\infty}(\Gamma): \forall \tau \in \mathscr{G}:\left.u\right|_{\tau} \in \mathbb{P}_{0}\right\}
$$


and, alternatively, we consider the space of continuous, piecewise linear functions

$$
S_{0,1}:=\left\{u \in C^{0}(\Gamma): \forall \tau \in \mathscr{G}:\left.\left(u \circ \chi_{\tau}\right)\right|_{\tau} \in \mathbb{P}_{1}\right\}
$$

for the space discretization. As a basis for $S_{-1,0}$, we choose the characteristic functions for the panels $\tau \in \mathscr{G}$, while the basis for $S_{0,1}$ consists of the standard hat functions, lifted to the surface $\Gamma$. The general notation is $S$ for the boundary element space and $\left(b_{i}\right)_{i=1}^{M}$ for the basis. The mesh width is given by

$$
h:=\max _{\tau \in \mathscr{G}} h_{\tau}, \quad \text { where } h_{\tau}:=\operatorname{diam} \tau .
$$

For the space-time discrete solution at time $t_{n}$, we employ the ansatz

$$
\phi_{\Delta t, h}^{n}(y)=\sum_{i=1}^{M} \phi_{n, i} b_{i}(y)
$$

where $\phi_{n}=\left(\phi_{n, i}\right)_{i=1}^{M} \in \mathbb{R}^{M}$ are the nodal values of the discrete solution at time step $t_{n}$. The collection of these solution vectors is denoted by $\vec{\phi}_{N}:=\left(\phi_{i}\right)_{i=0}^{N} \in \mathbb{R}^{(N+1) M}$. Note that we always include $\phi_{0}$ in this vector although it is always zero.

For the Galerkin boundary element method, we replace $\phi_{\Delta t}^{j}$ in (3.7) by some $\phi_{\Delta t, h}^{j} \in S$ and impose the integral equation not pointwise but in a weak form: Find $\phi_{\Delta t, h}^{n} \in S$ of the form (3.8) such that

$$
\sum_{j=0}^{n} \sum_{i=1}^{M} \phi_{j, i} \int_{\Gamma} \int_{\Gamma} \omega_{n-j}^{\Delta t}(x-y) b_{i}(y) b_{k}(x) \mathrm{d} \Gamma_{y} \mathrm{~d} \Gamma_{x}=\int_{\Gamma} g_{\Delta t}^{n}(x) b_{k}(x) \mathrm{d} \Gamma_{x}
$$

for all $1 \leqslant k \leqslant M$ and $n=1, \ldots, N$. This can be written as a linear system

$$
\sum_{j=0}^{n} \mathbf{A}_{n-j} \boldsymbol{\phi}_{j}=\mathbf{g}_{n}, \quad n=1, \ldots, N
$$

with

$$
\left(\mathbf{A}_{n}\right)_{k, i}:=\int_{\Gamma} \int_{\Gamma} \omega_{n}^{\Delta t}(x-y) b_{i}(y) b_{k}(x) \mathrm{d} \Gamma_{y} \mathrm{~d} \Gamma_{x}
$$

and

$$
\left(\mathbf{g}_{n}\right)_{k}=\int_{\Gamma} g_{\Delta t}^{n}(x) b_{k}(x) \mathrm{d} \Gamma_{x}
$$

\subsection{Algorithmic realization and sparse approximation}

The linear systems in (3.10) can be written in the compact block form

$$
\overrightarrow{\mathbf{A}}_{N} \overrightarrow{\boldsymbol{\phi}}_{N}:=\overrightarrow{\mathbf{g}}_{N}
$$


where the block matrix $\overrightarrow{\mathbf{A}}_{N} \in \mathbb{R}^{(N+1) M} \times \mathbb{R}^{(N+1) M}$ and the vector $\overrightarrow{\mathbf{g}}_{N} \in \mathbb{R}^{(N+1) M}$ are defined by

$$
\overrightarrow{\mathbf{A}}_{N}:=\left(\begin{array}{cccccc}
\mathbf{A}_{0} & \mathbf{0} & \cdots & & & \mathbf{0} \\
\mathbf{A}_{1} & \mathbf{A}_{0} & \ddots & & & \vdots \\
\mathbf{A}_{2} & \mathbf{A}_{1} & \ddots & & & \\
\vdots & \mathbf{A}_{2} & \ddots & & \ddots & \\
& & \ddots & \ddots & \ddots & \mathbf{0} \\
\mathbf{A}_{N} & \cdots & & \mathbf{A}_{2} & \mathbf{A}_{1} & \mathbf{A}_{0}
\end{array}\right) \text { and } \overrightarrow{\mathbf{g}}_{N}:=\left(\begin{array}{c}
\mathbf{g}_{0} \\
\mathbf{g}_{1} \\
\vdots \\
\mathbf{g}_{N}
\end{array}\right)
$$

The matrices $\mathbf{A}_{j}$ have dimension $M \times M$ and are fully populated. The straightforward procedure for solving this system is given by the following recursion.

For $n=1,2, \ldots$, one computes

$$
\mathbf{w}_{n}:=\mathbf{g}_{n}-\sum_{i=0}^{n-1} \mathbf{A}_{n-i} \boldsymbol{\phi}_{i}
$$

and then solves the system

$$
\mathbf{A}_{0} \phi_{n}=\mathbf{w}_{n}
$$

If we assume that a fast iterative procedure is employed which solves (3.14) in $\mathrm{O}\left(M^{2}\right)$ operations, the total amount of work is given by

$$
\underbrace{\mathrm{O}\left(N^{2} M^{2}\right)}_{(3.13)}+\underbrace{\mathrm{O}\left(N M^{2}\right)}_{(3.14)} .
$$

The quadratic growth of the computational complexity with respect to $N$ and $M$ would make the numerical solution of time domain boundary integral equations prohibitively expensive. Hence, a fast solution method of the block-triangular system (3.11) which is based on FFT has been proposed in Hairer et al. (1985). This reduces the computational complexity to $\mathrm{O}\left(M^{2} N \log ^{2} N\right)$ while the storage complexity is $\mathrm{O}\left(N M^{2}\right)$.

In this paper, we present an alternative method which avoids the use of FFT but employs a sparse approximation of the system matrices $\mathbf{A}_{n}$.

We recall the definition of the matrix $\mathbf{A}_{n}$,

$$
\left(\mathbf{A}_{n}\right)_{i, j}=\int_{\operatorname{supp}\left(b_{i}\right)} \int_{\operatorname{supp}\left(b_{j}\right)} \omega_{n}^{\Delta t}(x-y) b_{i}(x) b_{j}(y) \mathrm{d} \Gamma_{y} \mathrm{~d} \Gamma_{x},
$$

where $\operatorname{supp}\left(b_{i}\right)$ denotes the support of the basis function $b_{i}$. The matrices $\mathbf{A}_{n}$ are full matrices. However, it turns out that a substantial part of the matrix entries is small and can be replaced by zero. In Section 4.3, we derive that for the interval

$$
I_{n, \varepsilon}^{\Delta t}:=\left[t_{n}-c_{n, \varepsilon}^{\Delta t}, t_{n}+c_{n, \varepsilon}^{\Delta t}\right] \cap[0, \operatorname{diam} \Gamma],
$$

with

$$
c_{n, \varepsilon}^{\Delta t}=3 \sqrt{\Delta t} \sqrt{t_{n}} \log \frac{1}{\varepsilon},
$$


we have

$$
\left|\omega_{n}^{\Delta t}(d)\right| \leqslant \frac{\varepsilon}{4 \pi d} \quad \forall d \notin I_{n, \varepsilon}^{\Delta t} .
$$

Let $\mathscr{P}_{\varepsilon}^{n} \subset\{1, \ldots, M\} \times\{1, \ldots, M\}$ be defined by

$$
\mathscr{P}_{\varepsilon}^{n}:=\left\{(i, j): \exists(x, y) \in \operatorname{supp} b_{i} \cap \operatorname{supp} b_{j}:\|x-y\| \in I_{n, \varepsilon}^{\Delta t}\right\} .
$$

This induces a sparse approximation $\tilde{\mathbf{A}}_{n}$ by

$$
\left(\tilde{\mathbf{A}}_{n}\right)_{i, j}:= \begin{cases}\left(\mathbf{A}_{n}\right)_{i, j}, & \text { if }(i, j) \in \mathscr{P}_{\varepsilon}^{n} \\ 0, & \text { otherwise. }\end{cases}
$$

In summary, the space-time discretization with sparse matrix approximation is given by replacing the matrices $\mathbf{A}_{n}$ in (3.12) by the sparse versions (3.19) and plugging the corresponding solution $\left(\tilde{\boldsymbol{\phi}}_{0}, \tilde{\boldsymbol{\phi}}_{1}, \ldots, \tilde{\boldsymbol{\phi}}_{N}\right)^{\mathrm{T}}$ into the basis representation

$$
\tilde{\phi}_{\Delta t, h}^{n}:=\sum_{i=1}^{M} \tilde{\phi}_{n, i} b_{i} .
$$

The following procedure is the algorithmic formulation of our sparse method for solving (3.11).

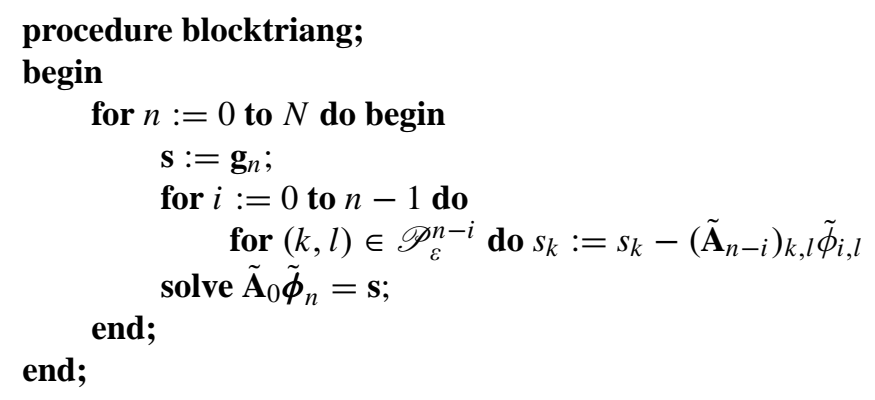

The solution of the system $\tilde{\mathbf{A}}_{0} \tilde{\boldsymbol{\phi}}_{n}=\mathbf{s}$ should be realized by means of an iterative solver which takes into account the sparsity of $\tilde{\mathbf{A}}_{0}$.

\section{Convergence analysis}

The convergence analysis consists of three parts. In Section 4.1, the analysis of the space-time discretization without sparse matrix approximation is given. The sparse approximation of the matrices $\mathbf{A}_{n}$ induces a perturbation in the space discretization and in Section 4.2, we analyse the effect of such perturbations on the overall discretization error. The size of the perturbation depends on the smallness of the function $\omega_{n}^{\Delta t}$ outside the interval $I_{n, \varepsilon}^{\Delta t}$. In Section 4.3, we determine the interval $I_{n, \varepsilon}^{\Delta t}$ such that the arising perturbation error is in balance with the overall discretization error.

\subsection{Error estimates for the space-time discretization without sparse matrix approximation}

For the semidiscrete solution $\phi_{\Delta t}^{n}$ of (3.2), the following theorem holds (Lubich, 1994). 
THEOREM 4.1 For smooth compatible data $g$, for $0 \leqslant \Delta t \leqslant \Delta t^{*}, \Delta t^{*}$ arbitrary, the error satisfies

$$
\left(\Delta t \sum_{n=0}^{N}\left\|\phi_{\Delta t}^{n}(\cdot)-\phi\left(\cdot, t_{n}\right)\right\|_{H^{-1 / 2}(\Gamma)}^{2}\right)^{1 / 2} \leqslant C_{\Delta t^{*}} \Delta t^{2}\|g\|_{H_{0}^{5}\left(0, T ; H^{1 / 2}(\Gamma)\right)} .
$$

The $A$-stability of the linear multistep method is inherited to the convolution quadrature method, i.e. all $\Delta t^{*}$ are permitted in the above estimate.

Let $\left(\phi_{k, h}^{n}\right)_{n=0}^{N}$ be the sequence of solutions of (3.9) at the time levels $t_{n}, n=0,1, \ldots, N$. We quote from Lubich (1994) the following convergence theorem.

THEOREM 4.2 For smooth compatible data $g$, the fully discrete method (3.9) (Galerkin in space, operational quadrature in time) is unconditionally stable and the solution $\phi_{\Delta t, h}^{n} \in S_{m-1, m}, 0 \leqslant n \leqslant N$, $m \in\{0,1\}$, satisfies the error estimate

$$
\left\|\phi\left(\cdot, t_{n}\right)-\phi_{\Delta t, h}^{n}(\cdot)\right\|_{H^{-1 / 2}(\Gamma)} \leqslant C_{g}\left(\Delta t^{2}+h^{m+\frac{3}{2}}\right) .
$$

As an immediate consequence of this theorem, we see that the spatial and temporal errors are balanced if

$$
\Delta t^{2} \sim h^{m+\frac{3}{2}}
$$

\subsection{Perturbations in the space discretization}

In this section, we study the influence of replacing the matrices $\mathbf{A}_{n}$ by the sparse approximation $\tilde{\mathbf{A}}_{n}$. Our perturbation analysis is based on the theory which was developed in Lubich (1994). For this, we introduce the time continuous, space discrete problem which is given by: Find $\phi_{h}:[0, T] \rightarrow S$ such that

$$
\int_{0}^{t} \int_{\Gamma} \int_{\Gamma} k(x-y, t-\tau) \phi_{h}(y, \tau) \psi_{h}(x) \mathrm{d} \Gamma_{y} \mathrm{~d} \Gamma_{x} \mathrm{~d} \tau=\int_{\Gamma} g(x, t) \psi_{h}(x) \mathrm{d} \Gamma_{x} \quad \forall \psi_{h} \in S .
$$

We recall the definition of the one-sided Laplace transform

$$
\hat{f}(s):=(\mathscr{L} f)(s):=\int_{0}^{\infty} \mathrm{e}^{-s t} f(t) \mathrm{d} t .
$$

(Convention: if a function depends on space and time variables, the Laplace transform is always applied to the time variable.) Applying this transformation to (4.2) and using the rule for the Laplace transform of convolutions, we obtain (cf. Lubich, 1994)

$$
\int_{\Gamma} \int_{\Gamma} \hat{k}(x-y, s) \hat{\phi}_{h}(y, s) \psi_{h}(x) \mathrm{d} \Gamma_{y} \mathrm{~d} \Gamma_{x}=\int_{\Gamma} \hat{g}(x, s) \psi_{h}(x) \mathrm{d} \Gamma_{x} \quad \forall \psi_{h} \in S, \quad \forall s \in I_{\sigma},
$$

where $I_{\sigma}:=\{\sigma+\mathrm{i} u: u \in \mathbb{R}\}$ for some $\sigma>0$. The Laplace transform of $k$ is given by

$$
\hat{k}(z, s)=\frac{\mathrm{e}^{-s\|z\|}}{4 \pi\|z\|}
$$


and $\hat{\phi}_{h}$ is the Laplace transform of $\phi_{h}$. For $s \in I_{\sigma}$, we define the operator $V_{h}(s): S \rightarrow S$ by

$$
\left(V_{h}(s) \varphi_{h}, \psi_{h}\right)_{L^{2}(\Gamma)}:=\int_{\Gamma} \int_{\Gamma} \hat{k}(x-y, s) \varphi_{h}(y) \overline{\psi_{h}}(x) \mathrm{d} \Gamma_{y} \mathrm{~d} \Gamma_{x} \quad \forall \varphi_{h}, \psi_{h} \in S .
$$

Let $P_{h}: H^{1 / 2}(\Gamma) \rightarrow S$ denote the orthogonal projection, i.e.

$$
\left(P_{h} f, \psi_{h}\right)_{L^{2}(\Gamma)}:=\left(f, \psi_{h}\right)_{L^{2}(\Gamma)} \quad \forall \psi_{h} \in S .
$$

With these notations at hand, the time continuous, spatially discrete problem (4.3) can be written in the compact form: Find $\hat{\phi}_{h}: I_{\sigma} \rightarrow S$ such that

$$
\left(V_{h}(s) \hat{\phi}_{h}(s), \psi_{h}\right)_{L^{2}(\Gamma)}=\left(P_{h} \hat{g}(\cdot, s), \psi_{h}\right)_{L^{2}(\Gamma)} \quad \forall \psi_{h} \in S, \quad \forall s \in I_{\sigma} .
$$

The time discretization can be described by replacing $s$ in $V_{h}(s)$ by $\gamma\left(\mathrm{e}^{-s \Delta t}\right) / \Delta t:$ Find $\hat{\phi}_{\Delta t, h}: I_{\sigma} \rightarrow S$ such that

$$
\left(V_{\Delta t, h}(s) \hat{\phi}_{\Delta t, h}(s), \psi_{h}\right)_{L^{2}(\Gamma)}=\left(P_{h} \hat{g}(\cdot, s), \psi_{h}\right)_{L^{2}(\Gamma)} \quad \forall \psi_{h} \in S, \quad \forall s \in I_{\sigma},
$$

where $V_{\Delta t, h}(s):=V_{h}\left(\gamma\left(\mathrm{e}^{-s \Delta t}\right) / \Delta t\right)$.

REMARK 4.3 The solution $\phi_{\Delta t, h}^{n}$ at time step $t_{n}=n \Delta t$ (cf. (3.8)) can be written by means of the inverse Laplace transform as

$$
\phi_{\Delta t, h}^{n}=\left(\mathscr{L}^{-1} \hat{\phi}_{\Delta t, h}\right)\left(t_{n}\right)
$$

Next, we express the solution $\tilde{\phi}_{\Delta t, h}^{n}$ of (3.20) in a similar fashion. Our cut-off strategy is based on the approximation of the coefficients $\omega_{n}^{\Delta t}(d)$ in the power series

$$
\hat{k}\left(d, \frac{\gamma(\zeta)}{\Delta t}\right)=\sum_{n=0}^{\infty} \omega_{n}^{\Delta t}(d) \zeta^{n}
$$

by

$$
\tilde{\omega}_{n}^{\Delta t}(d):= \begin{cases}\omega_{n}^{\Delta t}(d), & d \in I_{n, \varepsilon}^{\Delta t} \\ 0, & d \notin I_{n, \varepsilon}^{\Delta t}\end{cases}
$$

Let

$$
\begin{gathered}
G(d, s):=\hat{k}\left(d, \frac{\gamma\left(\mathrm{e}^{-s \Delta t}\right)}{\Delta t}\right)=\sum_{n=0}^{\infty} \omega_{n}^{\Delta t}(d) \mathrm{e}^{-s \Delta t n}, \\
\tilde{G}(d, s):=\sum_{n=0}^{\infty} \tilde{\omega}_{n}^{\Delta t}(d) \mathrm{e}^{-s \Delta t n} .
\end{gathered}
$$

For $s \in I_{\sigma}$, let $\tilde{V}_{\Delta t, h}(s): S \rightarrow S$ be the operator defined by

$$
\left(\tilde{V}_{\Delta t, h}(s) \varphi_{h}, \psi_{h}\right)_{L^{2}(\Gamma)}:=\int_{\Gamma} \int_{\Gamma} \tilde{G}(\|x-y\|, s) \varphi_{h}(y) \overline{\psi_{h}}(x) \mathrm{d} \Gamma_{y} \mathrm{~d} \Gamma_{x} \quad \forall \varphi_{h}, \psi_{h} \in S .
$$


Consider the problem: Find $\widehat{\tilde{\phi}}_{\Delta t, h}(s) \in S$ such that

$$
\left(\tilde{V}_{\Delta t, h}(s) \widehat{\tilde{\phi}}_{\Delta t, h}(s), \psi_{h}\right)_{L^{2}(\Gamma)}=\left(P_{h} \hat{g}(\cdot, s), \psi_{h}\right)_{L^{2}(\Gamma)} \quad \forall \psi_{h} \in S, \quad \forall s \in I_{\sigma}
$$

Then, the solution $\tilde{\phi}_{\Delta t, h}^{n}$ of (3.20) can be expressed by means of the inverse Laplace transform

$$
\tilde{\phi}_{\Delta t, h}^{n}:=\left(\mathscr{L}^{-1} \widehat{\tilde{\phi}}_{\Delta t, h}\right)\left(t_{n}\right)
$$

By combining (4.4) and (4.7), we see that the Laplace transform of the error $e_{\Delta t, h}:=\tilde{\phi}_{\Delta t, h}-\phi_{\Delta t, h}$ satisfies

$$
\left(V_{\Delta t, h}(s) \hat{e}_{\Delta t, h}(s), \psi_{h}\right)_{L^{2}(\Gamma)}=\left(\left(V_{\Delta t, h}(s)-\tilde{V}_{\Delta t, h}(s)\right) \widehat{\tilde{\phi}}_{\Delta t, h}(s), \psi_{h}\right)_{L^{2}(\Gamma)} \quad \forall \psi_{h} \in S, \quad \forall s \in I_{\sigma} .
$$

This leads to the estimate

$$
\left\|\hat{e}_{\Delta t, h}(s)\right\|_{H^{-1 / 2}(\Gamma)} \leqslant\left\|V_{\Delta t, h}^{-1}(s)\right\|_{H^{-1 / 2}(\Gamma) \leftarrow H^{1 / 2}(\Gamma)}\left\|\left(V_{\Delta t, h}(s)-\tilde{V}_{\Delta t, h}(s)\right) \widehat{\tilde{\phi}}_{\Delta t, h}(s)\right\|_{H^{1 / 2}(\Gamma)}
$$

for all $s \in I_{\sigma}$.

In order to estimate the terms in (4.8), we need the following estimate of $\left\|V^{-1}(s)\right\|_{H^{-1 / 2}(\Gamma) \leftarrow H^{1 / 2}(\Gamma)}$ (cf. Lubich, 1994, (2.20)): Let $\sigma>0$, then there exists $M(\sigma)$ such that

$$
\left\|V^{-1}(s)\right\|_{H^{-1 / 2}(\Gamma) \leftarrow H^{1 / 2}(\Gamma)} \leqslant M(\sigma)|s|^{2} \quad \forall \operatorname{Re}(s)>\sigma .
$$

LEMMA 4.4 Let the time discretization be based on convolution quadrature with the BDF2 scheme. Then, for $\sigma>0$ there exists $c_{\sigma}>0$ independent of the discretization parameters $\Delta t, h$ such that

$$
\left\|V_{\Delta t, h}^{-1}(s) P_{h}\right\|_{H^{-1 / 2}(\Gamma) \leftarrow H^{1 / 2}(\Gamma)} \leqslant c_{\sigma} \frac{1}{\Delta t^{2}} \quad \forall s \in I_{\sigma} .
$$

Proof. From Lubich (1994, (5.17)), we deduce the estimate

$$
\begin{aligned}
\left\|V_{\Delta t, h}^{-1}(s) P_{h}\right\|_{H^{-1 / 2}(\Gamma) \leftarrow H^{1 / 2}(\Gamma)} & =\left\|V_{h}^{-1}\left(\gamma\left(\mathrm{e}^{-s \Delta t}\right) / \Delta t\right) P_{h}\right\|_{H^{-1 / 2}(\Gamma) \leftarrow H^{1 / 2}(\Gamma)} \\
& \leqslant M\left(\sigma_{0}\right)\left|\frac{\gamma\left(\mathrm{e}^{-s \Delta t}\right)}{\Delta t}\right|^{2} \quad \forall s \in I_{\sigma},
\end{aligned}
$$

for $\sigma_{0}$ such that $\operatorname{Re}\left(\frac{\gamma\left(\mathrm{e}^{-s \Delta t}\right)}{\Delta t}\right)>\sigma_{0}$ for all $s \in I_{\sigma} . \sigma_{0}$ can be chosen independently of $\Delta t$. The estimate now follows due to the boundedness of $\left|\gamma\left(\mathrm{e}^{-s \Delta t}\right)\right|$.

Next, we turn to the second factor in the right-hand side of (4.8). For the following lemma, we need an inverse inequality which holds for our boundary element spaces (cf. Dahmen et al., 2004), while the constant depends on the quasiuniformity of the mesh. Let $C_{\mathrm{inv}}>0$ denote the smallest constant such that

$$
\left\|\psi_{h}\right\|_{L^{2}(\Gamma)} \leqslant C_{\mathrm{inv}} h^{-1 / 2}\left\|\psi_{h}\right\|_{H^{-1 / 2}(\Gamma)} \quad \forall \psi_{h} \in S
$$

holds. 
LEMMA 4.5 Let the time discretization be based on convolution quadrature with the BDF2 scheme. Then,

$$
\left\|\left(V_{\Delta t, h}(s)-\tilde{V}_{\Delta t, h}(s)\right) \widehat{\tilde{\phi}}_{\Delta t, h}(s)\right\|_{H^{1 / 2}(\Gamma)} \leqslant \frac{c_{\Delta} \varepsilon h^{-1}}{1-\mathrm{e}^{-\sigma \Delta t}}\left\|\widehat{\tilde{\phi}}_{\Delta t, h}(s)\right\|_{H^{-1 / 2}(\Gamma)} \quad \forall s \in I_{\sigma}
$$

The constant $c_{\Delta}$ is associated with the Laplace operator and $C_{\mathrm{inv}}$ is independent of the discretization parameters $\Delta t$ and $h$.

Proof. For any $\phi_{h} \in S$, the difference $\left(V_{\Delta t, h}(s)-\tilde{V}_{\Delta t, h}(s)\right) \hat{\phi}_{h}(s)$ can be written in the form

$$
\left\|\left(V_{\Delta t, h}(s)-\tilde{V}_{\Delta t, h}(s)\right) \hat{\phi}_{h}(s)\right\|_{H^{1 / 2}(\Gamma)}=\sup _{\substack{\varphi_{h} \in S \backslash\{0\} \\\left\|\varphi_{h}\right\|_{H^{-1 / 2}(\Gamma)}}}\left|\int_{\Gamma} \int_{\Gamma} \delta(\|x-y\|) \hat{\phi}_{h}(y, s) \varphi_{h}(x) \mathrm{d} \Gamma_{y} \mathrm{~d} \Gamma_{x}\right|,
$$

where (cf. (4.6))

$$
\delta(d):=\sum_{n=0}^{\infty}\left(\omega_{n}^{\Delta t}(d)-\tilde{\omega}_{n}^{\Delta t}(d)\right) \mathrm{e}^{-s \Delta t n}
$$

From the construction of our cut-off strategy (cf. (3.17)), we deduce that

$$
|\delta(d)| \leqslant \frac{\varepsilon}{4 \pi d} \sum_{n=0}^{\infty} \mathrm{e}^{-\sigma \Delta t n}=\frac{\varepsilon}{4 \pi d\left(1-\mathrm{e}^{-\sigma \Delta t}\right)} .
$$

By using the well-known $L^{2}$-continuity of the single-layer potential for the Laplacian, we obtain

$$
\begin{aligned}
\left\|\left(V_{\Delta t, h}(s)-\tilde{V}_{\Delta t, h}(s)\right) \hat{\phi}_{h}(s)\right\|_{H^{1 / 2}(\Gamma)} & \leqslant \frac{\varepsilon}{1-\mathrm{e}^{-\sigma \Delta t}} \sup _{\substack{\varphi_{h} \in S \backslash\{0\} \\
\left\|\varphi_{h}\right\|_{H^{-1 / 2}(\Gamma)}}} \int_{\Gamma} \int_{\Gamma} \frac{\left|\hat{\phi}_{h}(y, s) \| \varphi_{h}(x)\right|}{4 \pi\|x-y\|} \mathrm{d} \Gamma_{y} \mathrm{~d} \Gamma_{x} \\
& \leqslant \frac{C \varepsilon h^{-\frac{1}{2}}}{1-\mathrm{e}^{-\sigma \Delta t}}\left\|\phi_{h}\right\|_{L^{2}(\Gamma)} \\
& \leqslant \frac{c_{\Delta} \varepsilon h^{-1}}{1-\mathrm{e}^{-\sigma \Delta t}}\left\|\phi_{h}\right\|_{H^{-1 / 2}(\Gamma)} .
\end{aligned}
$$

REMARK 4.6 Note that the previous lemma holds under the more general assumption

$$
\left|\omega_{n}^{\Delta t}(d)-\tilde{\omega}_{n}^{\Delta t}(d)\right| \leqslant \frac{\varepsilon}{4 \pi d}
$$

Finally, we investigate the existence and boundedness of the solution $\tilde{\phi}_{\Delta t, h}$. We do not employ the possible smoothness of $\tilde{\phi}_{\Delta t, h}$ with respect to time since only the constants in the convergence and complexity estimates would be improved but not the rates. 
LEMMA 4.7 Let the time discretization be based on convolution quadrature with the BDF2 scheme. Then given $\sigma>0$, for all cut-off parameters $\varepsilon$ in (3.19) with $0<\varepsilon<\frac{1-\mathrm{e}^{-\sigma \Delta t}}{2 c_{\Delta} c_{\sigma}} h \Delta t^{2}$, the solution $\tilde{\phi}_{\Delta t, h}$ in (3.20) exists and satisfies the stability estimate

$$
\left\|\widehat{\tilde{\phi}}_{\Delta t, h}(s)\right\|_{H^{-1 / 2}(\Gamma)} \leqslant 2 c_{\sigma} \Delta t^{-2}\|\hat{g}(s)\|_{H^{1 / 2}(\Gamma)} \quad \forall s \in I_{\sigma} .
$$

Proof. We start with the splitting

$$
\tilde{V}_{\Delta t, h}(s)=V_{\Delta t, h}(s)(I-X(s)) \quad \text { with } X(s):=V_{\Delta t, h}^{-1}(s)\left(V_{\Delta t, h}(s)-\tilde{V}_{\Delta t, h}(s)\right) .
$$

Lemmata 4.4 and 4.5 imply

$$
\|X(s)\|_{H^{-1 / 2}(\Gamma) \leftarrow H^{-1 / 2}(\Gamma)} \leqslant c_{\Delta} c_{\sigma} \frac{1}{\Delta t^{2}} \frac{\varepsilon h^{-1}}{1-\mathrm{e}^{-\sigma \Delta t}} .
$$

By choosing $0<\varepsilon<\frac{1-\mathrm{e}^{-\sigma \Delta t}}{2 c_{\Delta} c_{\sigma}} h \Delta t^{2}$, we obtain $\|X(s)\|_{H^{-1 / 2}(\Gamma) \leftarrow H^{-1 / 2}(\Gamma)}<1 / 2$ uniformly for all $s \in I_{\sigma}$. This directly implies the stability estimate

$$
\left\|\tilde{V}_{\Delta t, h}^{-1}(s) P_{h}\right\|_{H^{1 / 2}(\Gamma) \leftarrow H^{-1 / 2}(\Gamma)} \leqslant 2\left\|V_{\Delta t, h}^{-1}(s) P_{h}\right\|_{H^{1 / 2}(\Gamma) \leftarrow H^{-1 / 2}(\Gamma)} \leqslant 2 c_{\sigma} \Delta t^{-2} .
$$

The combination of Lemmata 4.4, 4.5 and 4.7 leads to the convergence estimate of the solution $\tilde{\phi}_{\Delta t, h}$.

THEOREM 4.8 Let the time discretization be based on convolution quadrature with the BDF2 scheme. We assume that the exact solution $\phi(\cdot, t)$ is in $H^{m+1}(\Gamma)$ for any $t \in[0, T]$. Then, for all cut-off parameters $\varepsilon$ in (3.19) with $0<\varepsilon<\frac{1-\mathrm{e}^{-\sigma \Delta t}}{2 c_{\Delta} c_{\sigma}} h \Delta t^{2}$, the solution $\tilde{\phi}_{\Delta t, h}$ in (3.20) exists and satisfies the error estimate

$$
\left\|\tilde{\phi}_{\Delta t, h}^{n}-\phi\left(\cdot, t_{n}\right)\right\|_{H^{-1 / 2}(\Gamma)} \leqslant C_{g}\left(t_{n}\right)\left(\varepsilon h^{-1} \Delta t^{-5}+\Delta t^{2}+h^{m+\frac{3}{2}}\right),
$$

where $C_{g}$ depends on the right-hand side $g$ and on $\sigma$.

Proof. We employ the splitting

$$
\tilde{\phi}_{\Delta t, h}^{n}-\phi\left(t_{n}\right)=e_{\Delta t, h}^{n}+\left(\phi_{\Delta t, h}^{n}-\phi\left(t_{n}\right)\right) .
$$

The estimate (Lubich, 1994, Theorem 5.4) implies, for the second summand,

$$
\left\|\phi_{\Delta t, h}^{n}-\phi\left(t_{n}\right)\right\|_{H^{-1 / 2}(\Gamma)} \leqslant C h^{m+\frac{3}{2}} .
$$

The first summand can be estimated by combining Lemmata 4.4, 4.5 and 4.7

$$
\begin{aligned}
\left\|\hat{e}_{\Delta t, h}(s)\right\|_{H^{-1 / 2}(\Gamma)} & \leqslant 2 c_{\sigma}^{2} c_{\Delta} h^{-1} \Delta t^{-4} \frac{\varepsilon}{1-\mathrm{e}^{-\sigma \Delta t}}\|\hat{g}(\cdot, s)\|_{H^{1 / 2}(\Gamma)} \\
& \leqslant C_{\sigma} \varepsilon h^{-1} \Delta t^{-5}\|\hat{g}(\cdot, s)\|_{H^{1 / 2}(\Gamma)} \quad \forall s \in I_{\sigma} .
\end{aligned}
$$

From this, the estimate of the perturbation $\tilde{\phi}_{\Delta t, h}-\phi_{\Delta t, h}$ in the original time space follows from the Laplace inversion formula. 
COROLlary 4.9 Let the assumptions as in Theorem 4.8 be satisfied. Let

$$
\Delta t^{2} \sim h^{m+\frac{3}{2}}
$$

and choose

$$
\varepsilon \sim h^{\frac{7 m}{2}+\frac{25}{4}}
$$

Then, the solution $\tilde{\phi}_{\Delta t, h}^{n}$ exists and converges with optimal rate

$$
\left\|\tilde{\phi}_{\Delta t, h}^{n}-\phi\left(\cdot, t_{n}\right)\right\|_{H^{-1 / 2}(\Gamma)} \leqslant C_{g}\left(t_{n}\right) h^{m+\frac{3}{2}} \sim C_{g}\left(t_{n}\right) \Delta t^{2} .
$$

\subsection{Approximation of $\omega_{n}$ by cut-off}

In this section, we analyse the approximation of the convolution functions

$$
\omega_{n}^{\Delta t}(d)=\left.\frac{1}{n !} \frac{\partial^{n}}{\partial \zeta^{n}} \frac{\mathrm{e}^{-\gamma(\zeta) \frac{d}{\Delta t}}}{4 \pi d}\right|_{\zeta=0}
$$

where

$$
\gamma(\zeta)=\frac{1}{2}\left(\zeta^{2}-4 \zeta+3\right)
$$

We recall the explicit formula as in (3.6)

$$
\omega_{n}^{\Delta t}(d)=\frac{1}{n !} \frac{1}{4 \pi d}\left(\frac{d}{2 \Delta t}\right)^{n / 2} \mathrm{e}^{-\frac{3 d}{2 \Delta t}} H_{n}\left(\sqrt{\frac{2 d}{\Delta t}}\right),
$$

where $H_{n}$ are the Hermite polynomials. For $n=0$, we have

$$
\omega_{0}^{\Delta t}(d)=\frac{\mathrm{e}^{-\frac{3}{2} \frac{d}{\Delta t}}}{4 \pi d}
$$

with a singularity at $d=0$, and for $n=1$,

$$
\omega_{1}^{\Delta t}(d)=\frac{1}{\Delta t} \frac{\mathrm{e}^{-\frac{3}{2} \frac{d}{\Delta t}}}{2 \pi} .
$$

In Fig. 1, we plot $\omega_{n}^{\Delta t}(d)$ for $\Delta t=1$ and different $n$. The convolution functions are approximately scaled and translated versions of each other. To find an estimate for $\omega_{n}^{\Delta t}(d)$, we employ the ansatz

$$
\left|\omega_{n}^{\Delta t}(d)\right| \leqslant \frac{1}{4 \pi d} \sigma_{n} \Omega_{n}\left(f_{n}^{\Delta t}(d)\right)
$$

with some scaling factors $\sigma_{n}$, some translation functions $f_{n}^{\Delta t}(d)$ and a function $\Omega_{n}(x)$ that converges towards a function $\Omega(x)$ as $n \rightarrow \infty$. 


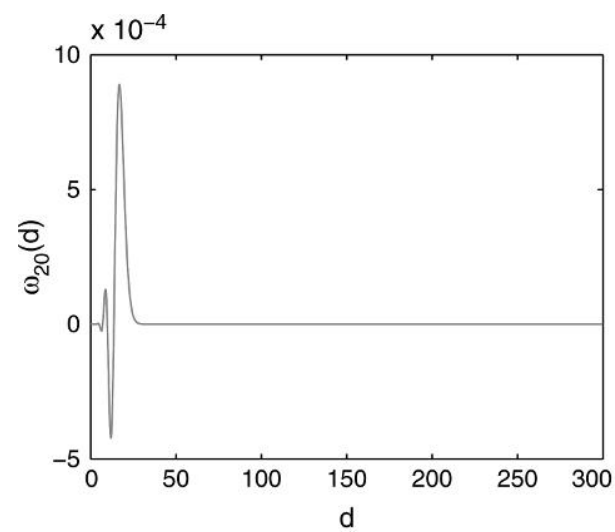

(a) $n=20$

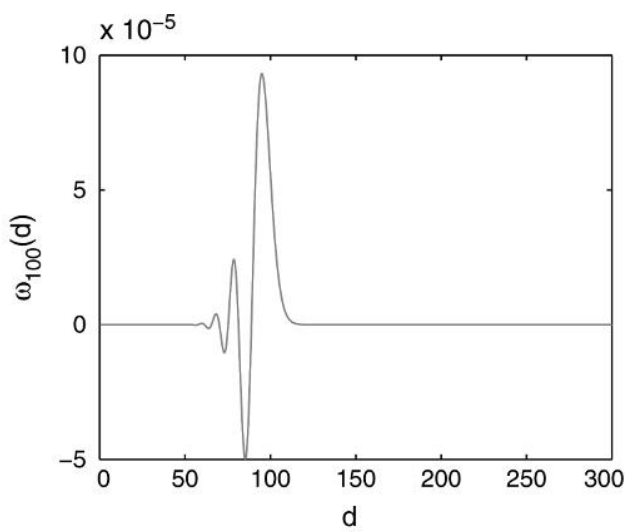

(b) $n=100$

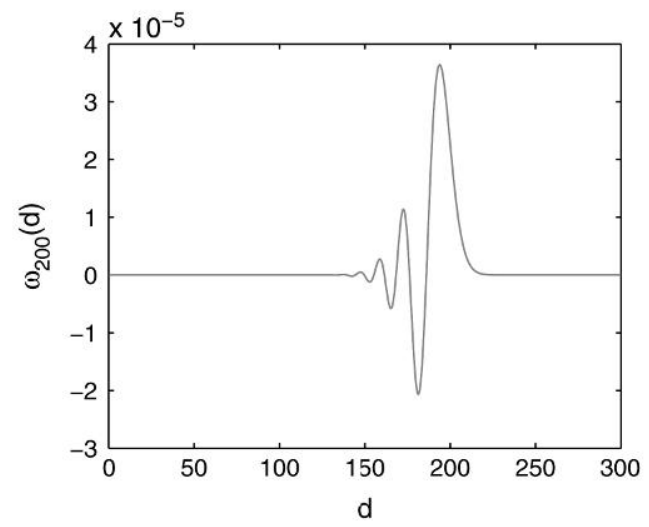

(c) $n=200$

FIG. 1. The convolution weights $\omega_{n}^{\Delta t}(d)$ for $\Delta t=1$ and different values of $n$.

LEMMA 4.10 For $n \geqslant 1$, let

$$
\Omega_{n}(x)=\left(\frac{x}{\sqrt{n}}+1\right)^{n / 2} \mathrm{e}^{-\frac{x \sqrt{n}}{2}} \quad \text { and } \quad \sigma_{n}=\frac{k}{(2 \pi n)^{\frac{1}{4}}}
$$

with $k \approx 1.086435$. Then,

$$
\left|\omega_{n}^{\Delta t}(d)\right| \leqslant \frac{1}{4 \pi d} \sigma_{n} \Omega_{n}\left(\frac{d-t_{n}}{\sqrt{\Delta t} \sqrt{t_{n}}}\right) .
$$

Proof. To obtain an estimate for $\left|\omega_{n}^{\Delta t}(d)\right|$, we use the following estimate (cf. Abramowitz \& Stegun, 1972, (22.14.17)):

$$
\left|H_{n}(x)\right|<\mathrm{e}^{x^{2} / 2} k 2^{n / 2} \sqrt{n !}
$$


with $k \approx 1.086435$. Applying this to (4.15) yields

$$
\left|\omega_{n}^{\Delta t}(d)\right| \leqslant \frac{k}{4 \pi d} \frac{\left(\frac{d}{\Delta t}\right)^{n / 2} \mathrm{e}^{-\frac{d}{2 \Delta t}}}{\sqrt{n !}} .
$$

For $n \geqslant 1$, Stirling's formula leads to $\frac{1}{\sqrt{n !}} \leqslant \frac{\left(\frac{e}{n}\right)^{n / 2}}{(2 \pi n)^{\frac{1}{4}}}$ and we obtain

$$
\left|\omega_{n}^{\Delta t}(d)\right| \leqslant \frac{k}{4 \pi d} \frac{\mathrm{e}^{n / 2}\left(\frac{d}{t_{n}}\right)^{n / 2}}{(2 \pi n)^{\frac{1}{4}}} \mathrm{e}^{-\frac{d}{2 \Delta t}}=\frac{k}{4 \pi d} \frac{1}{(2 \pi n)^{\frac{1}{4}}} \Omega_{n}\left(\frac{d-t_{n}}{\sqrt{\Delta t} \sqrt{t_{n}}}\right) .
$$

This estimate is illustrated in Fig. 2.

LEMMA 4.11 There holds

$$
\lim _{n \rightarrow \infty} \Omega_{n}(x)=\mathrm{e}^{-x^{2} / 4} .
$$

Proof. The logarithm of $\Omega_{n}$ can be written as

$$
\begin{aligned}
\log \Omega_{n}(x) & =\frac{n}{2} \log \left(1+\frac{x}{\sqrt{n}}\right)-\frac{x \sqrt{n}}{2} \\
& =\frac{n}{2} \sum_{k=1}^{\infty} \frac{(-1)^{k+1}}{k}\left(\frac{x}{\sqrt{n}}\right)^{k}-\frac{x \sqrt{n}}{2} \\
& =-\frac{1}{4} x^{2}+\sum_{k=3}^{\infty} \frac{1}{3} \frac{(-1)^{k+1}}{2 k}\left(\frac{x^{k}}{n^{\frac{k}{2}-1}}\right),
\end{aligned}
$$

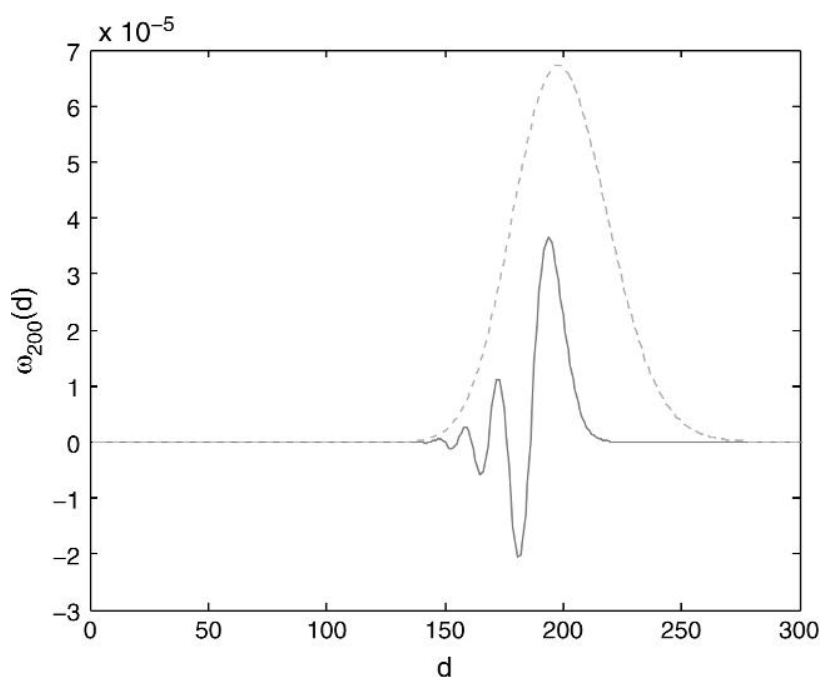

FIG. 2. Comparison of $\omega_{100}^{1}(d)$ (solid line) and $\frac{1}{4 \pi d} \sigma_{n} \Omega_{n}$ (dashed line). 
from which we conclude that

$$
\lim _{n \rightarrow \infty} \log \Omega_{n}(x)=-\frac{1}{4} x^{2}
$$

holds. Thus, the statement of the lemma follows.

REMARK 4.12 For $x \geqslant 0, \Omega_{n}(x)$ is decreasing for increasing $n$. For $-\sqrt{n} \leqslant x \leqslant 0, \Omega_{n}(x)$ is increasing for increasing $n$.

In Section 3.3, we have introduced a sparse approximation of $\mathbf{A}_{n}$ by replacing $\omega_{n}^{\Delta t}(d)$ by zero outside an interval $I_{n, \varepsilon}^{\Delta t}=\left[t_{n}-c_{n, \varepsilon}^{\Delta t}, t_{n}+c_{n, \varepsilon}^{\Delta t}\right]$. To determine $I_{n, \varepsilon}^{\Delta t}$ such that

$$
\left|\omega_{n}^{\Delta t}(d)\right| \leqslant \frac{\varepsilon}{4 \pi d} \quad \forall d \notin I_{n, \varepsilon}^{\Delta t}
$$

we first seek an interval $\tilde{I}_{n, \varepsilon}$ such that

$$
\Omega_{n}(x) \leqslant C \varepsilon \quad \forall x \notin \tilde{I}_{n, \varepsilon} .
$$

Simple analysis shows that $\Omega_{n}$ has one maximum at $x=0$ and is strictly monotonously increasing for $x<0$ and strictly monotonously decreasing for $x>0$. Due to Remark 4.12, sufficient conditions for $\Omega_{n}(x) \leqslant C \varepsilon$ are $\Omega_{1}(x)=\sqrt{x+1} \mathrm{e}^{-\frac{1}{2} x} \leqslant C \varepsilon$ for positive $x$ and $\lim _{n \rightarrow \infty} \Omega_{n}(x)=\mathrm{e}^{-\frac{x^{2}}{4}} \leqslant C \varepsilon$ for negative $x$. If we choose

$$
\tilde{c}=3 \log \frac{1}{\varepsilon},
$$

inequality (4.16) is satisfied for all $x \notin \tilde{I}_{n, \varepsilon}:=[-\tilde{c}, \tilde{c}]$ with $C=\sqrt{3} \mathrm{e}^{-1 / 3}$.

LEMMA 4.13 Let $n \geqslant 1$ and $c_{n, \varepsilon}^{\Delta t}=\sqrt{\Delta t} \sqrt{t_{n}} \tilde{c}$ with $\tilde{c}$ as in (4.17). For $I_{n, \varepsilon}^{\Delta t}:=\left[t_{n}-c_{n, \varepsilon}^{\Delta t}, t_{n}+c_{n, \varepsilon}^{\Delta t}\right]$, there holds

$$
\left|\omega_{n}^{\Delta t}(d)\right| \leqslant \frac{\varepsilon}{4 \pi d} \quad \forall d \notin I_{n, \varepsilon}^{\Delta t} .
$$

For $n=0$ and $I_{0, \varepsilon}^{\Delta t}:=\left[0, \frac{2}{3} \Delta t \log \frac{1}{\varepsilon}\right]$, there holds

$$
\left|\omega_{0}^{\Delta t}(d)\right| \leqslant \frac{\varepsilon}{4 \pi d} \quad \forall d \notin I_{0, \varepsilon}^{\Delta t} .
$$

Proof. We have

$$
\left|\omega_{n}^{\Delta t}(d)\right| \leqslant \frac{k}{4 \pi d} \frac{1}{(2 \pi n)^{\frac{1}{4}}} \Omega_{n}\left(\frac{d-t_{n}}{\sqrt{\Delta t} \sqrt{t_{n}}}\right) \leqslant \frac{\varepsilon}{4 \pi d}
$$

since $\frac{d-t_{n}}{\sqrt{\Delta t} \sqrt{t_{n}}} \notin[-\tilde{c}, \tilde{c}]$ and $\frac{k}{(2 \pi n)^{\frac{1}{4}}} \sqrt{3} \mathrm{e}^{-1 / 3}<1$. For $n=0$, we have

$$
\omega_{0}^{\Delta t}(d)=\frac{\mathrm{e}^{-\frac{3}{2} \frac{d}{\Delta t}}}{4 \pi d}
$$

and the condition $d \geqslant \frac{2}{3} \Delta t \log \frac{1}{\varepsilon}$ implies

$$
\frac{\mathrm{e}^{-\frac{3}{2} \frac{d}{d t}}}{4 \pi d} \leqslant \frac{\varepsilon}{4 \pi d}
$$




\section{Complexity estimates}

First, we determine the storage requirements for the matrices $\tilde{\mathbf{A}}_{n}$. For the boundary element mesh, we assume that the dimension of the boundary element space satisfies

$$
c_{1} h^{-2} \leqslant M \leqslant C_{1} h^{-2} .
$$

A further assumption is related to the surface $\Gamma$ and the mesh $\mathscr{G}$. We assume that there is a moderate constant $C$ such that for any $1 \leqslant i \leqslant M$, the subset

$$
\mathscr{P}_{i}^{n}:=\left\{j \in\{1, \ldots, M\}:(i, j) \in \mathscr{P}_{\varepsilon}^{n}\right\},
$$

with $\mathscr{P}_{\varepsilon}^{n}$ as in (3.18), satisfies

$$
\sharp \mathscr{P}_{i}^{n} \leqslant C \max \left\{1, \frac{\sqrt{\Delta t} t_{n}^{\frac{3}{2}}}{h^{2}} \log M\right\} .
$$

This assumption can be derived from two assumptions, namely, that the area of

$$
R_{i, n}:=\left\{y \in \Gamma: \exists x \in \operatorname{supp} b_{i}:\|x-y\| \in I_{n, \varepsilon}^{\Delta t}\right\}
$$

satisfies $\left|R_{i, n}\right| \leqslant C \sqrt{\Delta t} t_{n}^{\frac{3}{2}}|\log (\varepsilon)|$ and that $c h^{2} \leqslant \operatorname{supp} b_{j} \leqslant C h^{2}$. Choosing $\varepsilon$ for the cut-off such that it is balanced with the discretization error, we have

$$
|\log \varepsilon| \sim \log M .
$$

THEOREM 5.1 The number of nonzero entries in the sparse approximation $\tilde{\mathbf{A}}_{n}$ is bounded from above by

$$
\sharp \text { nonzero entries } \leqslant C M \max \left\{1, N^{-\frac{1}{2}} t_{n}^{\frac{3}{2}} M \log M\right\} .
$$

Proof. The number of nonzero matrix entries in $\tilde{\mathbf{A}}$ can be estimated by using (5.2),

$$
\sum_{i=1}^{M} \sharp \mathscr{P}_{i}^{n} \leqslant C M \max \left\{1, \sqrt{\Delta t} t_{n}^{\frac{3}{2}} h^{-2} \log M\right\} .
$$

The theorem immediately follows when replacing $\Delta t$ and $h$ by $N^{-1}$ and $M^{-\frac{1}{2}}$, respectively.

Using relation (4.1), the following result is obtained.

COROLlARY 5.2 Under the assumption (4.1), the number of nonzero entries in the sparse approximation $\tilde{\mathbf{A}}_{n}$ is bounded from above

- for piecewise constant boundary elements by

$$
C t_{n}^{\frac{3}{2}} M^{1+\frac{13}{16}} \log M .
$$

For the first time steps, $t_{n}=q \Delta t$, where $q=\mathrm{O}(\log M)$, we obtain the improved upper bound

$$
C M^{1+\frac{1}{4}} \log ^{\frac{5}{2}} M \text {. }
$$


- for piecewise linear boundary elements by

$$
C t_{n}^{\frac{3}{2}} M^{1+\frac{11}{16}} \log M
$$

For the first time steps, $t_{n}=q \Delta t$, where $q=\mathrm{O}(\log M)$, the improved upper bound is

$$
C M \text {. }
$$

Note that the solution of (3.11) requires that $N$ linear systems of the form

$$
\mathbf{A}_{0} \phi_{n}=\text { r.h.s. }
$$

have to be solved. If the dimension $M$ is large, iterative methods have to be employed for this purpose which require a matrix-vector multiplication in each iteration step. In this light, the improved estimates (5.4b) and (5.4d) of the number of nonzero matrix entries for $\tilde{\mathbf{A}}_{0}$ accelerate this solution process.

Next, we will discuss the computational complexity for procedure blocktriang from Section 3.3.

THEOREM 5.3 The number of arithmetic operations needed in procedure blocktriang is bounded by

$$
\sharp \text { operations } \leqslant C N^{\frac{3}{2}} M^{2} \log M \text {. }
$$

Proof. By using the estimate (5.3), the number of arithmetic operations for performing the nested loop over $i, j, k, \ell$ in procedure blocktriang can be estimated by

$$
\begin{aligned}
2 \sum_{i=0}^{N} \sum_{j=0}^{i-1} \sharp \mathscr{P}_{\varepsilon}^{i-j} & \leqslant 2 \sum_{i=0}^{N} \sum_{j=0}^{i-1} \sum_{\ell=1}^{M} \sharp \mathscr{P}_{\ell}^{i-j} \leqslant C M \sum_{i=0}^{N} \sum_{j=0}^{i-1} \max \left\{1, N^{-\frac{1}{2}} t_{i-j}^{\frac{3}{2}} M \log M\right\} \\
& \leqslant C M \sum_{j=0}^{N-1} \sum_{\ell=1}^{N-j} \max \left\{1, \ell^{\frac{3}{2}} N^{-2} M \log M\right\} \\
& \leqslant C M N \max \left\{N, N^{\frac{1}{2}} M|\log M|\right\} \\
& \leqslant C N^{\frac{3}{2}} M^{2} \log M .
\end{aligned}
$$

Note that the matrix $\mathbf{A}_{0}$ is positive definite (cf. Sauter \& Schwab, 2004, Theorem 3.5.4) and its condition number behaves like $h^{-1}$ (cf. Sauter \& Schwab, 2004, Section 4.5). From Sauter \& Schwab (2004, Theorem 6.1.7), we deduce that $\mathrm{O}\left(h^{-\frac{1}{2}} \log h\right)$ iterations of a cg-algorithm suffice to compute a solution so that the overall convergence rate is preserved. Due to the sparsity of $\mathbf{A}_{0}$ (cf. Theorem 5.3), the amount of work is given by

$$
C\left(h^{-\frac{1}{2}} \log h\right) N M^{1+\frac{1-m}{4}} \log M \leqslant C N \begin{cases}M^{1+\frac{1}{2}} \log ^{2} M, & m=0, \\ M^{1+\frac{1}{4}} \log ^{2} M, & m=1 .\end{cases}
$$

Due to (4.1), this is bounded by the operations necessary for the nested loop. Thus, the assertion follows. 


\section{Conclusions}

In this paper, we have followed the convolution quadrature approach by Lubich and combined it with a Galerkin boundary element method for solving the retarded potential boundary integral formulation of the wave equation. We have presented a simple a priori cut-off strategy where the number of matrix elements which have to be computed is substantially reduced and a significant portion of the matrix is replaced by zero. A perturbation analysis established the stability of the perturbed problem.

The analysis in this paper paves the way for further complexity reductions. In Hackbusch et al. (2007) and Banjai \& Sauter (2007), we develop a variant of the panel clustering method for the wave equation in order to further reduce the storage requirements and also reduce the computational costs.

\section{Acknowledgement}

Thanks are due to the anonymous referees for their helpful comments and suggestions.

\section{REFERENCES}

Abramowitz, M. \& Stegun, I. (1972) Handbook of Mathematical Functions. Applied Mathematics Series 55. Washington, DC: National Bureau of Standards, U.S. Department of Commerce.

BAMBERgER, A. \& HA-DuONG, T. (1986) Formulation variationelle espace-temps pour le calcul par potentiel retardé d'une onde acoustique. Math. Methods Appl. Sci., 8, 405-435, 598-608.

BANJAI, L. \& SAUTER, S. (2007) Rapid solution of the wave equation in unbounded domains. Technical Report 10/07/2007. University of Zurich (to appear).

Birgisson, B., Siebrits, E. \& Pierce, A. (1999) Elastodynamic direct boundary element methods with enhanced numerical stability properties. Int. J. Numer. Methods Eng., 46, 871-888.

BLUCK, M. \& WALKER, S. (1996) Analysis of three-dimensional transient acoustic wave propagation using the boudary integral equation method. Int. J. Numer. Methods Eng., 39, 1419-1431.

Ciarlet, P. (1987) The Finite Element Method for Elliptic Problems. Amsterdam: North-Holland.

Costabel, M. (1994) Developments in boundary element methods for time-dependent problems. Problems and Methods in Mathematical Physics (L. Jentsch \& F. Tröltsch eds). Leipzig: B.G. Teubner, pp. 17-32.

Dahmen, W., Faermann, B., Graham, I., Hackbusch, W. \& Sauter, S. (2004) Inverse inequalities on non-quasiuniform meshes and applications to the mortar element method. Math. Comput., 73, 1107-1138.

DAVIES, P. (1994) Numerical stability and convergence of approximations of retarded potential integral equations. SIAM J. Numer. Anal., 31, 856-875.

DAVIES, P. (1997) Averaging techniques for time marching schemes for retarded potential integral equations. Appl. Numer. Math., 23, 291-310.

DAVIES, P. \& DUNCAN, D. (2004) Stability and convergence of collocation schemes for retarded potential integral equations. SIAM J. Numer. Anal., 42, 1167-1188.

Ding, Y., Forestier, A. \& HA-DuONG, T. (1989) A Galerkin scheme for the time domain integral equation of acoustic scattering from a hard surface. J. Acoust. Soc. Am., 86, 1566-1572.

Ergin, A., Shanker, B. \& Michielssen, E. (2000) Fast analysis of transient acoustic wave scattering from rigid bodies using the multilevel plane wave time domain algorithm. J. Acoust. Soc. Am., 117, 1168-1178.

Friedman, M. \& SHAW, R. (1962) Diffraction of pulses by cylindrical obstacles of arbitrary cross section. J. Appl. Mech., 29, 40-46.

Gradshteyn, I. S. \& RyZhiK, I. (1965) Table of Integrals, Series, and Products. New York: Academic Press.

HACKBusch, W. (1992) Elliptic Differential Equations. Berlin: Springer.

Hackbusch, W., Kress, W. \& Sauter, S. (2007) Sparse convolution quadrature for time domain boundary integral formulations of the wave equation by cutoff and panel-clustering. Boundary Element 
Analysis: Mathematical Aspects and Applications (M. Schanz \& O. Steinbach eds). Springer Lecture Notes in Applied and Computational Mechanics, 113-134.

HA-DuOng, T. (2003) On retarded potential boundary integral equations and their discretization. Computational Methods in Wave Propagation (M. Ainsworth, P. Davies, D. Duncan, P. Martin \& B. Rynne eds), vol. 31. Heidelberg: Springer, pp. 301-336.

Ha-Duong, T., Ludwig, B. \& Terrasse, I. (2003) A Galerkin BEM for transient acoustic scattering by an absorbing obstacle. Int. J. Numer. Methods Eng., 57, 1845-1882.

Hairer, E., Lubich, C. \& SChlichte, M. (1985) Fast numerical solution of nonlinear Volterra convolution equations. SIAM J. Sci. Stat. Comput., 6, 532-541.

KRESS, W. \& SAUTER, S. (2006) Numerical treatment of retarded boundary integral equations by sparse panel clustering. Technical Report 17-2006. Universität Zürich. Available at http://www.math.unizh.ch/fileadmin/ math/preprints/17-06.pdf.

Lubich, C. (1988a) Convolution quadrature and discretized operational calculus I. Numer. Math., 52, 129-145.

LUBICH, C. (1988b) Convolution quadrature and discretized operational calculus II. Numer. Math., 52, 413-425.

LUBICH, C. (1994) On the multistep time discretization of linear initial-boundary value problems and their boundary integral equations. Numer. Math., 67, 365-389.

Lubich, C. \& SCHNEIDER, R. (1992) Time discretization of parabolic boundary integral equations. Numer. Math., 63, 455-481.

MCLEAn, W. (2000) Strongly Elliptic Systems and Boundary Integral Equations. Cambridge: Cambridge University Press.

MiLler, E. (1987) An overview of time-domain integral equations models in electromagnetics. J. Electromagn. Waves Appl., 1, 269-293.

Rynne, B. \& SMith, P. (1990) Stability of time marching algorithms for the electric field integral equation. J. Electromagn. Waves Appl., 4, 1181-1205.

SAUter, S. \& SchWAB, C. (2004) Randelementmethoden. Leipzig: Teubner. 